\title{
COMPARACIÓN DE MODELOS NO LINEALES PARA DESCRIBIR CURVAS DE CRECIMIENTO EN LACABRA CATALANA
}

\author{
Comparison of Nonlinear Models to Describe Growth Curves in the \\ Catalan Goat
}

Pere M. Parés-Casanova ${ }^{1,2}$, Irina Kucherova

\section{Resumen}

\begin{abstract}
El presente trabajo tuvo por objetivo estudiar las curvas de crecimiento de machos y hembras de la población «Cabra Catalana». Se evaluó la capacidad de ajuste de tres modelos de crecimiento (Logístico, von Bertalanffy y Gompertz) a los datos de peso de 77 animales (21 machos y 56 hembras de diversas edades). Los modelos presentaron valores diferentes en cuanto a los criterios de bondad de ajuste, donde el modelo logístico tuvo una mejor descripción de las curvas de crecimiento en los machos, y el de von Bertalanffy para las hembras. Aun así, el modelo logístico tiende a sobrestimar el peso inicial de los animales.
\end{abstract}

Palabras clave: modelo logístico, modelo von Bertalanffy, modelo Gompertz, ontogenia

\section{AbStract}

The aim of this study was to evaluate the growth curves for males and females in the Catalan goat population using different non lineal models (Logistic, von Bertalanffy and Gompertz). The models were applied to the study of body weight of 77 animals (21 males and 56 females of various ages). Fitness was different for each model where the logistic model was the best for males and the von Bertalanffy for females. Still, the logistic model tends to overestimate the initial weight of the animals.

Key words: logistic model, von Bertalanffy model, Gompertz model, ontogeny

${ }^{1}$ Departament de Producció Animal, Universitat de Lleida, Av. Alcalde Rovira Roure, 191. E 25198

Lleida, Catalunya, España

${ }^{2}$ E-mail: peremiquelp@prodan.udl.cat

Recibido: 13 de enero de 2014

Aceptado para publicación: 3 mayo de 2014 


\section{INTRODUCCIÓN}

Los modelos matemáticos son ecuaciones que, entre otras cosas, permiten construir curvas continuas de una variable biológica en función de otra. Esta información es importante para efectos de investigación y recomendaciones de orden zootécnico [p.ej. buscando precocidad y mejor calidad de la canal (Souza y Bianchini, 1994)]. Diversos modelos han sido utilizados para describir la relación entre la edad del animal, su velocidad de crecimiento y madurez, pero dado que el crecimiento animal no sigue una tendencia lineal, es necesario explorar modelos no lineales que permitan estudiar el crecimiento animal.

La relación entre el cambio de una dimensión y el tiempo puede expresarse mediante las curvas generales de crecimiento. Como es sabido, estas curvas tienen una forma de tipo sigmoideo cuando el incremento de la dimensión se manifiesta en términos absolutos. Los modelos matemáticos utilizados para modelar el crecimiento animal tienen forma sigmoidal, pudiéndose diferenciar en ellos las siguientes fases: 1. Fase de aceleración, que idealmente debe tener su origen en el punto (0.0), y se caracteriza por una velocidad de crecimiento muy rápida y positiva llegando al máximo en el punto de inflexión de la curva; 2. Fase de desaceleración, en la que a partir del punto de inflexión la tasa de crecimiento comienza a disminuir; y 3. Fase lineal, en la que el animal deja de crecer o el crecimiento puede ser considerado como una mera reposición de tejidos (Priestley, 1988).

Los modelos Logístico, de Gompertz y de von Bertalanffy son algunas de las funciones de crecimiento frecuentemente utilizadas para describir el crecimiento de plantas, animales y órganos (Priestley, 1988). El requisito básico es obtener una expresión que dé el tamaño (en longitud o en peso) de los animales a una edad determinada cualquiera, donde esa expresión debe estar de acuerdo con los datos observados sobre tamaños o pesos a ciertas edades, y debe tener una forma matemática que pueda ser incorporada con suficiente facilidad en el modelo elegido. Estrictamente, la mayoría de los análisis zootécnicos están más enfocados en las tasas de crecimiento ponderal; es decir, en el aumento en peso por unidad de tiempo, que en el tamaño a diferentes edades. Esto se debe en parte a que muchos de los problemas que se plantean en la evaluación de las ganaderías son esencialmente problemas de comparación del peso del ganado, más que de la talla.

Son clásicas las aplicaciones de estos métodos en investigaciones pesqueras (Von Bertalanffy, 1951), aunque se han utilizado también en el estudio del crecimiento de dimensiones ponderales y lineales de mamíferos (Priestley, 1988). El modelo Logístico se caracteriza usualmente por la forma sigmoidal. La ecuación de Gompertz es también sigmoidal, pero más flexible debido al punto de inflexión. La ecuación de Von Bertalanffy es muy utilizada para describir el comportamiento animal (Goodall y Sprevak, 1984). Estos modelos presentan tres parámetros con interpretación biológica y una constante matemática (e). El parámetro $a$ corresponde al peso asintótico o peso adulto a la madurez; el parámetro $b$ es denominado parámetro de integración; y el parámetro $c$ corresponde a la estimativa de precocidad de madurez (Nobre et al., 1987), y cuanto mayor sea el valor de este parámetro, más precoz es el animal y viceversa (Brown et al., 1976).

La Cabra Catalana es una raza propia de Cataluña (NE de España), que se creía extinguida durante el primer tercio del siglo pasado. No obstante ello, en 2011 se localizaron unos pocos ejemplares caprinos adultos que respondían marcadamente a la raza. A raíz de ello, dentro del proyecto «Cultures Trobades» («Slow Food Terres de Lleida»), se adquirieron 21 animales (19 hembras y 2 machos), que pasaron a formar el núcleo fundacional, localizado actualmente en 
Cuadro 1. Descripción matemática de los modelos no lineales de crecimiento considerados en el estudio

\begin{tabular}{lcl}
\hline Modelo & N. ${ }^{\circ}$ de parámetros & Expresión matemática \\
\hline Logístico & 3 & $\mathrm{y}=\mathrm{a} /\left(1+\mathrm{be} \mathrm{c}^{-\mathrm{cx}}\right)$ \\
Gompertz & 3 & $\mathrm{y}=\mathrm{a}\left(\mathrm{b}^{\mathrm{cx}}\right)$ \\
von Bertalanffy & 3 & $\mathrm{y}=\mathrm{a}\left(1-\mathrm{be}^{-\mathrm{cx}}\right)$ \\
\hline
\end{tabular}

$\mathrm{y}=$ peso del animal en el tiempo

$\mathrm{a}=$ estimativa del peso a la madurez

$\mathrm{b}=$ parámetro de integración, no posee significado biológico

$\mathrm{c}=$ estimativa de precocidad de madurez

Vilanova de Meià, Cataluña. El sistema de explotación es totalmente extensivo, donde los animales aprovechan los pastos pobres en terreno abrupto, y soportando un clima extremo, por lo que es una población perfectamente adaptada al medio en que vive.

El objetivo de este trabajo fue evaluar los modelos matemáticos Logístico, von Bertalanffy y Gompertz en cuanto a su capacidad para describir el crecimiento de la Cabra Catalana.

\section{Material y Métodos}

Se utilizaron 21 machos y 56 hembras de la población caprina Cabra Catalana, de todas las edades (rango: nacimiento a más de 3 años), y procedentes del núcleo fundacional. Se registró la edad en base a las declaraciones del ganadero, y el peso vivo se obtuvo mediante el pesado directo de los animales con balanza digital. Los datos fueron recabados por la misma persona. Cuatro de los machos eran castrados; sin embargo, no se observaron diferencias significativas de peso en el análisis comparativo entre los machos enteros y los castrados (Mann-Whitney $\mathrm{U}=18, \mathrm{p}=0.16$ ), por lo que sus datos fueron agrupados con los datos de los machos enteros para el análisis estadístico.
Los modelos utilizados para describir el crecimiento de los animales fueron el Logístico, el de Gompertz y el de von Bertalanffy. Para este último, aunque algunos autores, como Fitzhugh (1976) lo presentan para peso como a $\left(1-b e^{-c x}\right)^{3}$, el modelo usado fue el originalmente descrito por von Bertalanffy (1951), a fin de hacer comparativos los tres modelos elegidos. Las expresiones matemáticas que representan a cada uno de los modelos y el número de parámetros se presentan en el Cuadro 1.

Los model os fueron ajustados a la edad de cada animal utilizando el procedimiento para modelizaciones no lineales del programa PAST (Hammer et al., 2010). Para distinguir la capacidad de ajuste de cada uno de los modelos en estudio se utilizó el criterio de información de Akaike (AIC), que combina teoría de máxima verosimilitud, información teórica y entropía de información (Motulsky y Christopoulos, 2003), considerando el mejor modelo aquel de menor valor AIC. Los intervalos de confianza se establecieron al 95\% mediante 1999 réplicas bootstrap.

La comparación del modelo según sexo se realizó con un análisis de covarianza (ANCOVA). Dado que la covariable (edad) debe poseer una relación lineal con la variable respuesta (peso vivo estimado, según el 
modelo elegido), se hizo la transformación logarítmica de los datos para convertir la relación en lineal. Finalmente, para evaluar el patrón de distribución de las diferencias entre los valores observados y los estimados, se recurrió al test $\mathrm{W}$ de normalidad de Shapiro-Wilk.

\section{Resultados y Discusión}

El comportamiento gráfico de los pesos vivos, en función de la edad en meses, para ambos sexos, aparece en la Fig. 1. Los animales crecieron de forma lineal hasta aproximadamente los 66 meses (800 días), estabilizando su crecimiento a partir de este momento y hasta los 3 años o más.

Los valores AIC de los modelos en estudio son presentados en el Cuadro 2. En los machos, el menor valor de AIC fue observado para el modelo logístico, con un AIC = 422.6, mientras que para las hembras el mejor modelo fue el de von Bertalanffy, con un $\mathrm{AIC}=2536.6$.

En las Figs. 2 y 3 se presentan las curvas de crecimiento observadas y predichas por el modelo logístico y de von Bertalanffy para animales machos y hembras, respectivamente. El test ANCOVA reflejó que existían diferencias significativas de crecimiento entre sexos $(\mathrm{F}=19.91, \mathrm{p}<0.001)$ (Fig. 4).

Además de los criterios matemáticos que permiten determinar la capacidad de ajuste de un modelo frente a otro, se requiere tener en cuenta la coherencia biológica de los parámetros estimados de cada modelo como parámetro de evaluación. En el Cuadro 3 se presentan los valores de los parámetros ecuacionales y la amplitud de la variación de cada uno de los tres parámetros de las curvas individuales de crecimiento para machos y hembras, después de su ajuste al modelo Logístico y de von Bertalanffy, respectivamente.
Cuadro 2. Valores del criterio de información de Akaike (AIC) en tres modelos matemáticos con valores de crecimiento de la Cabra Catalana

\begin{tabular}{llc}
\hline Modelo & Sexo & AIC \\
\hline Logístico & Machos & 422.6 \\
& Hembras & 4623.3 \\
Gompertz & Machos & 561.6 \\
& Hembras & 3272.1 \\
von Bertalanffy & Machos & 4834.1 \\
& Hembras & 2536.6 \\
\hline
\end{tabular}

El modelo logístico reveló que los machos presentan un ajuste mucho mejor que las hembras para el modelo de von Bertalanffy. Aunque los machos adultos presentaron un mayor peso que las hembras (67.9 vs. $54.5 \mathrm{~kg}$ ), la estimativa del peso maduro (parámetro $a$ ) fue similar para ambos sexos. El parámetro $c$ representa la tasa de madurez del animal e indica la velocidad de crecimiento en el sentido de alcanzar el peso asintótico a partir del peso inicial. Cuanto mayor el valor de $c$, más rápidamente el animal va a alcanzar su peso adulto. Animales con un alto valor de $c$ presentan madurez precoz en comparación con individuos de valores más bajos de $c$ y de peso inicial semejante. Las tasas de crecimiento expresadas en gramos por día, estimadas por el modelo, son mayores para machos que para hembras, siendo una diferencia apreciable. Con base a esto, se deduce que los machos crecen a una tasa mayor que las hembras, alcanzando a la misma edad, un peso adulto mayor.

La regresión lineal por el método de los mínimos cuadrados ordinario mostró un rango promedio de variación entre \pm 2.5 y \pm 5.1 $\mathrm{kg}$ de diferencia, entre los valores observados y los estimados, para machos y hembras respectivamente, con 95\% de confianza (Fig. 


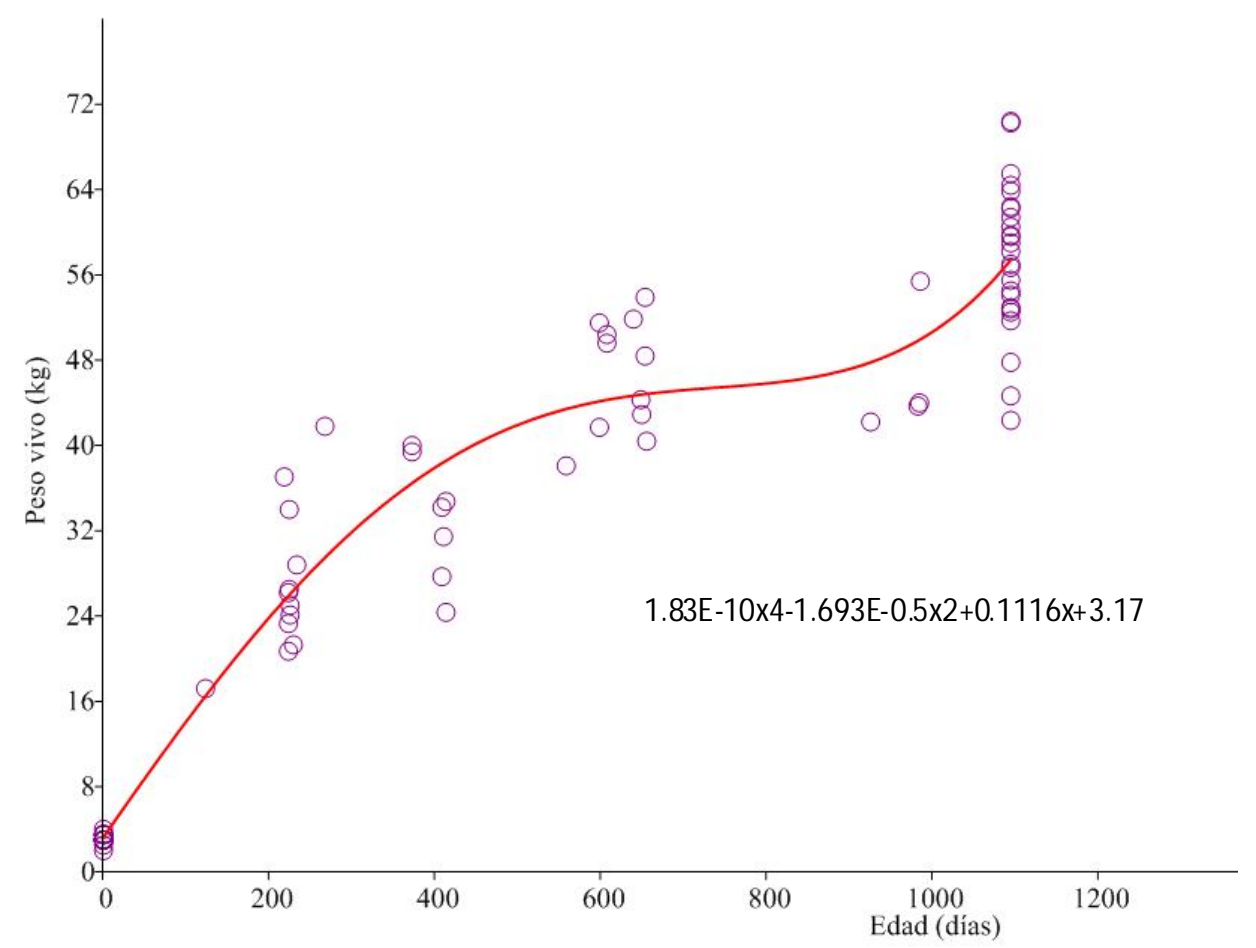

Figura 1. Comportamiento del peso vivo de la Cabra Catalana, de ambos sexos, desde el nacimiento hasta pasados los 3 años (1095 días)

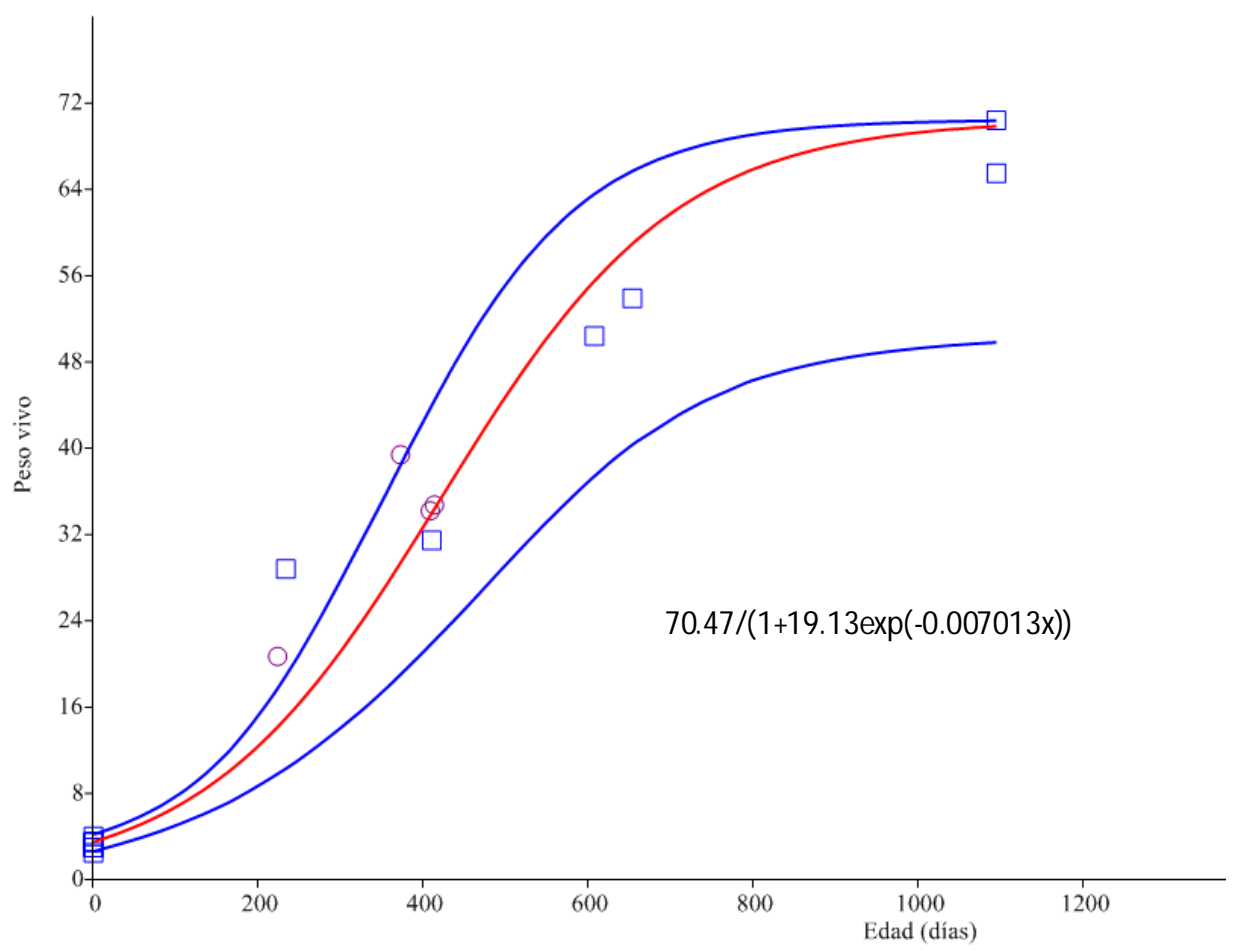

Figura 2. Curvas de crecimiento observadas y predichas para cabras catalanas machos (según el modelo logístico) $(\mathrm{n}=21)$. Los trazos externos indican el intervalo de confianza del 95\%. Peso vivo expresado en kilogramos. Los cuadrados corresponden a machos enteros y los círculos a los machos castrados 


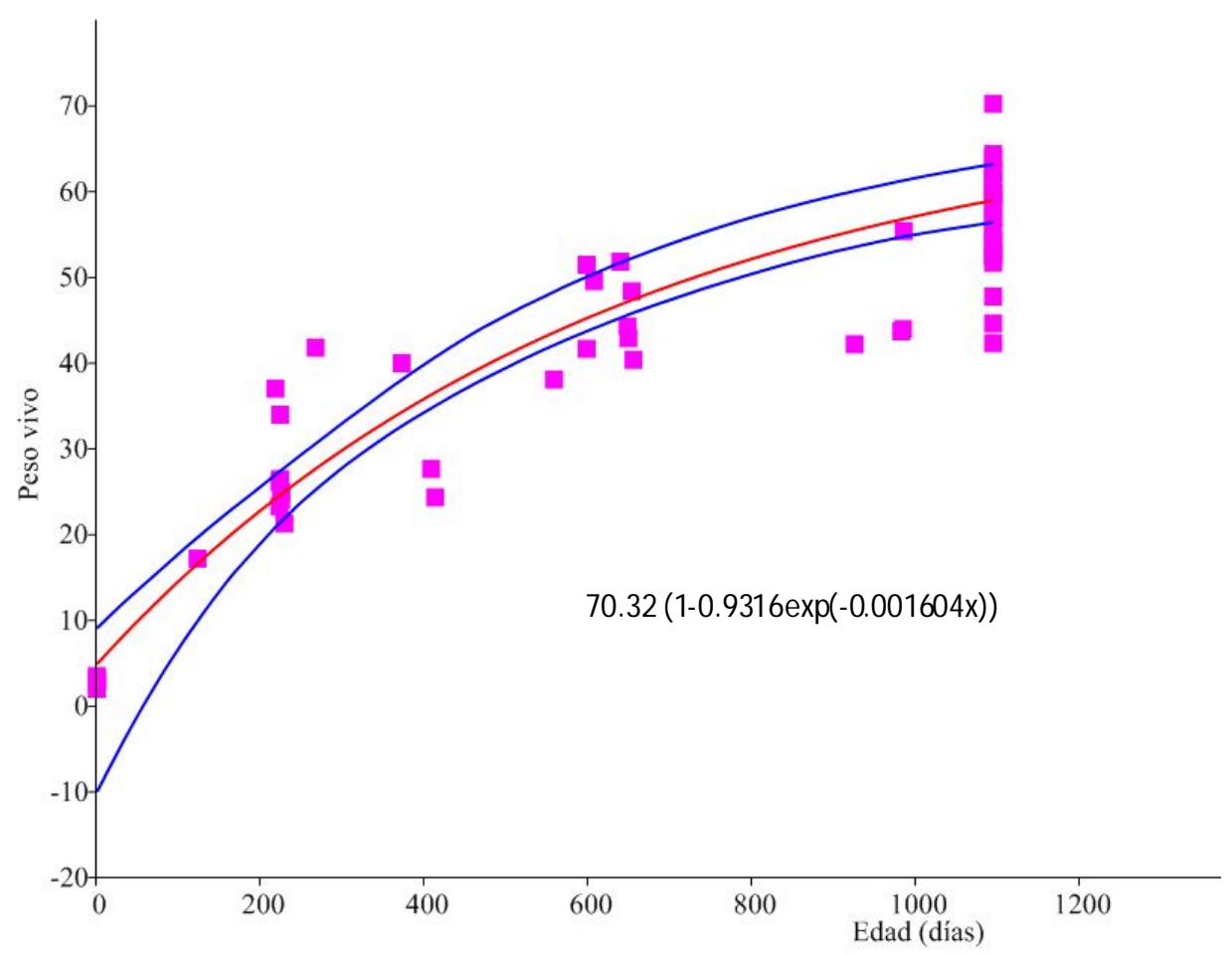

Figura 3. Curvas de crecimiento observadas y predichas para para cabras catalanas hembras (según el modelo de von Bertalanffy) ( $\mathrm{n}=56)$. Los trazos externos indican el intervalo de confianza del $95 \%$. Peso vivo expresado en kilogramos

5). El comportamiento de las diferencias fue de no normalidad ( $\mathrm{W}=0.960, \mathrm{p}=0.01)$. Este comportamiento no normal, o sea, aleatorio, y la variabilidad presente entre animales no justifican la determinación de un modelo estocástico, pues existe una componente de error aleatoria asociada al desarrollo del animal, pero posiblemente asociada también a las características genéticas, sobre todo en animales que son hijos de diferentes progenitores.

Según Hammon (1932, 1966), cuando un organismo crece se operan dos tipos de fenómenos: a) el incremento en peso y volumen en el transcurso del tiempo, y b) la modificación de las proporciones de las diversas regiones morfológicas, órganos y sistemas, hasta que se accede al estado adulto o estable. Para la Cabra Catalana, ambos sexos se ajustan a un patrón de crecimiento diferente, con un aumento general del peso mayor para los machos. La interpretación de los parámetros de ambos modelos se corresponde con valores lógicos, por cuanto, el parámetro $a=70.5 \mathrm{~kg}$ es una estimación del valor asintótico del animal (o de la población analizada). Según Brown et al. (1976), no representa necesariamente el mayor peso que el animal consigue, sino el peso medio al que se tiende en la madurez, independientemente de las variaciones estacionales. El valor de $b$ $=19.1$ para los machos y de 0.9 para las hembras es un parámetro importante que representa la tasa de madurez del animal en cada punto de la curva e indica la velocidad de crecimiento para alcanzar el peso asintótico. En muchas ocasiones, este valor se expresa en porcentaje y representa la velocidad de crecimiento. Por último, el parámetro $c<0.01$ está relacionado con el comportamiento de ambos modelos en los periodos iniciales. 


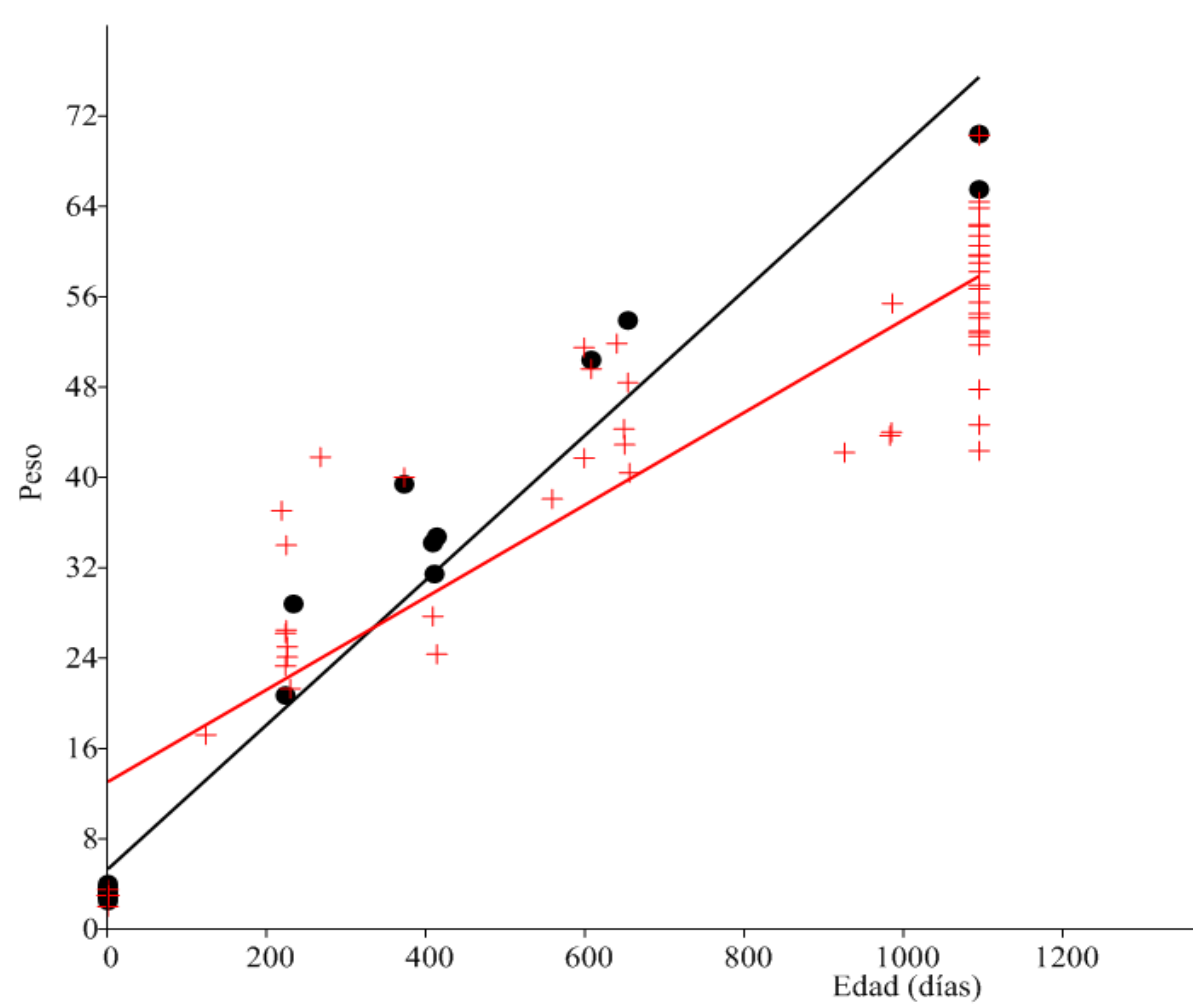

Figura 4. Comparación de los crecimientos lineales (datos transformados logarítmicamente) entre cabras catalanas machos $(n=21)$ y hembras $(n=56)$. Se observan diferencias significativas de crecimiento entre sexos $(\mathrm{F}=19.91, \mathrm{p}<0.001)$. Los círculos corresponden a machos y las cruces a las hembras

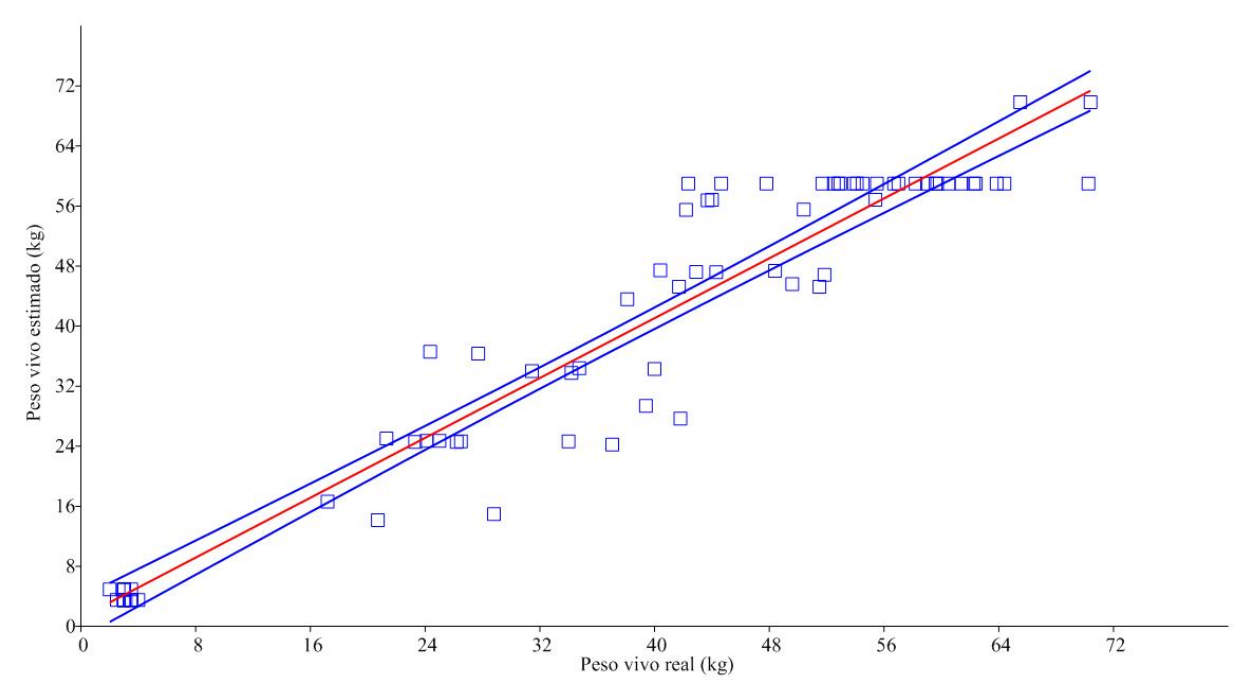

Figura 5. Regresión lineal por el método de los mínimos cuadrados ordinarios, para ambos sexos, en cabras catalanas $(n=77)$, donde la suma de cuadrados de las distancias verticales entre el peso vivo real y el peso vivo estimado, según el modelo elegido (modelo logístico para los machos machos [cuadrados vacíos] y von Bertalanffy para las hembras [cuadrados rellenos]) se ha minimizado. Los trazos externos indican el intervalo de confianza del $95 \%$ 
Según de Paz (2002), quien utilizó estos modelos en bovinos, el modelo logístico fue el más adecuado. Freitas (2005) concluyó que el modelo logístico y de Von Bertalanffy fueron los mejores para ajustar datos de crecimiento de ocho especies distintas. Además de los criterios matemáticos que permiten determinar la capacidad de ajuste de un modelo frente a otro, es necesario tener en cuenta como parámetro de evaluación la coherencia biológica de los parámetros estimados del modelo, criterio de evaluación que depende del conocimiento que tenga el investigador del fenómeno biológico en estudio, de la $\mathrm{Ca}$ bra Catalana, en este caso.

El grado de dimorfismo sexual depende de muchos factores (Frayer y Wolpoff, 1985), pero uno de los principales factores se relaciona a la nutrición proteico-calórica, que en caso de ser inadecuada actúa reduciendo las diferencias en tamaño entre sexos (Pucciarelli, 1980). En casos de estrés nutricional, y por lo menos en la especie humana, los hombres son más susceptibles y reducen su tamaño, mientras que las mujeres son más estables (Stini, 1969). Entonces, las diferencias en el desarrollo de la Cabra Catalana se podrían explicar por unas condiciones nutricionales subóptimas de las hembras. Análisis histológicos y osteológicos, fuera del alcance de este estudio, podrían aportar mayor información sobre esta hipótesis.

\section{Agradecimientos}

Es de obligado agradecimiento a Artur Bòria y Gerard Batalla, componentes del grupo «Cultures Trobades» ( «Slow Food Terres de Lleida»), que amablemente han permitido el trabajo con sus animales.

\section{Literatura Citada}

\section{Brown JE, Fitzhugh HA, Cartwright}

TC. 1976. A comparison of nonlinear models for describing weight-age relationships in cattle. J Anim Sci 42: 810-818.
2. Frayer DW, Wolpoff MH. 1985. Sexual dimorphism. Annu Rev Anthropol 14: 429-473.

3. Goodall EA, Sprevak D. 1984. A note on a stochastic model to describe the milk yield of a dairy cow. Anim Prod 38: 133136.

4. Fitzhugh HA. 1976. Analysis of growth curves and strategies for altering their shape. J Anim Sci 42: 1036-1051.

5. Freitas AR. 2005. Curvas de crescimento na produção animal. R Bras Zootec 34: 786-795.

6. Hammer $\emptyset$, Harper DAT, Ryan PD. 2001. PAST: Paleontological statistics software package for education and data analysis. v. $2.17 \mathrm{c}$. Palaeontologia Electronica 4(1): 9 p. [Internet]. Disponible en: http://palaeo-electronica.org/ 2001_1/past/issue1_01.htm

7. Hammond J. 1932. Growth and the development of mutton qualities in the sheep. London: Oliver \& Boyd. 597 p.

8. Hammond J. 1966. Principios de la explotación animal: reproducción, crecimiento y herencia. Zaragoza: Acribia. 363 p.

9. Motulsky H, Christopoulos A. 2003. Fitting models to biological data using linear and nonlinear regression: a practical guide to curve fitting. San Diego CA, USA: GraphPad Software. 351 p. [Internet]. Disponible en: http:// www.mcb5068. wustl.edu/MCB/ Lecturers/Baranski/Articles/ RegressionBook.pdf

10. Nobre PRC, Rosa A, Silva LO, Evangelista SR. 1987. Curvas de crescimento de gado Nelore ajustadas para diferentes freqüências de pesagens. Pesqui Agropecu Bras 22: 1027-1037.

11. Paz de C. 2002. Associação entre polimorfismos genéticos e parâmetros da curva de crescimento em bovinos de corte. Tese de Doutorado. Piracicaba, Brasil: Universidade de São Paulo. 89 p.

12. Priestley MB. 1988. Non-linear and non-stationary time series analysis. London: Academic Press. 237 p. 
13. Pucciarelli HM. 1980. The effect of race, sex and nutrition on craniofacial differentiation in rats. A multivariate analysis. Am J Phys Anthropol 53: 359-368.

14. Souza JC, Bianchini E. 1994. Estimativa do peso de bovinos de corte, aos 24 meses, da raça Nelore, usando curvas de crescimento. Rev Soc Bras Zootec 23: 85-91.
15. Stini WA. 1969. Nutritional stress and growth: sex difference in adaptive response. Am J Phys Anthropol 31: 417426.

16. von Bertalanffy L. 1951. Theoretische Biologie, Zweiter Band: Stoffwechsel, Wachstum. Bern, Switzerland: A Francke. 418 p. 